\title{
Prognostic factors of long-term outcome in surgically resectable pancreatic neuroendocrine tumors: A 12-year experience from a single center
}

\author{
BO ZHOU, JIXUAN DUAN, SHENG YAN, JIE ZHOU and SHUSEN ZHENG \\ Division of Hepatobiliary and Pancreatic Surgery, Department of Surgery, First Affiliated Hospital, \\ School of Medicine, Zhejiang University, Hangzhou, Zhejiang 310003, P.R. China
}

Received September 12, 2015; Accepted November 21, 2016

DOI: $10.3892 / \mathrm{ol} .2017 .5561$

\begin{abstract}
The present study was performed to assess the prognostic factors with respect to patient survival and clinicopathological characteristics for patients who underwent surgery for pancreatic neuroendocrine tumors (PNETs). A retrospective review of patients pathologically diagnosed with PNETs at the First Affiliated Hospital, Zhejiang University School of Medicine (Hangzhou, Zhejiang, China) between September 2002 and September 2013 was conducted. The clinicopathological findings, overall survival (OS) rate and disease-free survival (DFS) rate of the patients with PNETs were analyzed. Prognostic factors were determined by univariate and multivariate analyses. A total of 104 patients were selected. The median age at presentation was 52 years (range, 19-76 years). The most common surgical procedure was distal pancreatectomy (51.0\%), followed by pancreaticoduodenectomy (27.9\%) and local resection of the pancreas (16.3\%). The majority of the tumors were of low or moderate grade $(93.3 \%$, grade 1 or 2$)$ and were local or regional stage $(92.3 \%)$. The 1-, 3- and 5-year OS rates were 95,85 and $73 \%$, respectively. Meanwhile, the $1-, 3$ - and 5-year DFS rates were 86, 72 and 68\%, respectively. The OS and DFS rates were significantly different with regard to the grade (grades 1, 2 and 3; $\mathrm{P}<0.001$ ) and stage (local, regional and distant disease; $\mathrm{P}<0.001)$. Furthermore, patients with a low Ki-67 index experienced superior OS and DFS rates compared with patients with a high Ki-67 index $(\mathrm{P}<0.001)$. The presence of lymph node metastasis was predictive of inferior
\end{abstract}

Correspondence to: Professor Shusen Zheng, Division of Hepatobiliary and Pancreatic Surgery, Department of Surgery, First Affiliated Hospital, School of Medicine, Zhejiang University, 79 Qingchun Road, Hangzhou, Zhejiang 310003, P.R. China E-mail: shusenzheng@zju.edu.cn

Abbreviations: PNETs, pancreatic neuroendocrine tumors; OS, overall survival; DFS, disease-free survival

Key words: pancreatic neuroendocrine tumors, prognosis, grade, stage, lymph node metastasis, Ki-67 index, survival
OS and DFS rates compared with the absence of lymph node metastasis $(\mathrm{P}<0.001)$. OS rate for PNETs was significantly affected by resection margin status $(\mathrm{P}<0.001)$. In multivariable analysis, the significant factors associated with OS rate were stage $(\mathrm{P}=0.02)$, grade $(\mathrm{P}<0.001)$, lymph node status $(\mathrm{P}=0.025)$, Ki-67 index $(\mathrm{P}=0.031)$ and resection margin status $(\mathrm{P}<0.001)$. Meanwhile, stage $(\mathrm{P}=0.001)$, grade $(\mathrm{P}=0.017)$, lymph node status $(\mathrm{P}=0.02)$ and $\mathrm{Ki}-67$ index $(\mathrm{P}=0.016)$ were prognostic factors for DFS rate. In conclusion, grade, stage, Ki-67 index and lymph node involvement are significant prognostic factors for OS and DFS rates in surgically resectable PNETs. Furthermore, surgical margin status may also be an independent predictor for the prognosis of PNETs. It is anticipated that these findings may provide useful predictors of clinical survival, particularly in the setting of resected disease.

\section{Introduction}

Pancreatic neuroendocrine tumors (PNETs) are a heterogeneous group of neoplasms with varying clinical findings, and are considered as more indolent tumors with higher long-term survival rates than tumors of the exocrine pancreas (1-3). PNETs account for 1-2\% of all pancreatic neoplasms and 7.0\% of all neuroendocrine tumors (4). PNETs in the United States currently have an estimated annual incidence ranging between 2 and 5 cases per million individuals, but this appears to be increasing (5). PNETs can be classified as either functional or non-functional. The majority of PNETs (60-90\%) are non-functional (6).

It has been suggested that a complete surgical resection of a PNET is the only potentially curative treatment; this is similar to the suggestion for pancreatic adenocarcinoma. However, unlike pancreatic adenocarcinoma, surgical resection of the tumor and metastases can be performed where the entire tumor and metastatic disease can be removed, or surgery can be performed for palliation in patients with PNETs (6). The choice of surgical procedure, including enucleation, middle pancreatectomy, distal pancreatectomy and splenectomy, spleen-preserving distal pancreatectomy, pancreaticoduodenectomy or total pancreatectomy, depends on the tumor size and location (7). Subsequent to an R0 resection, the 5-year survival rate has been recorded at $86.4 \%$, 
but this rate decreases significantly following an incomplete resection (8).

Although a considerable amount of research has been undertaken, our understanding of the natural history and predictors of survival for PNETs remains incomplete. Several studies reported that the main factors predicting survival after the resection of PNETs were the tumor grade, the presenting symptoms, the size of the lesion, lymph node involvement and the presence of metastases (4,9-14). To evaluate the experience of a single institution with regard to these uncommon tumors, a retrospective review of 104 consecutive resections of PNETs was conducted in the present study in order to analyze clinical characteristics, to assess long-term survival following surgical treatment, and to discuss the prognosis of affected patients.

\section{Patients and methods}

Patients. A total of 104 patients who underwent surgical resection for PNETs between September 2002 and September 2013 at the First Affiliated Hospital, Zhejiang University School of Medicine (Hangzhou, Zhejiang, China) were retrospectively reviewed.

Methods. The diagnosis of PNET was made based on standard histological criteria (Fig. 1). Hematoxylin and eosin (HE) staining and immunohistochemical staining for chromogranin A (dilution, 1:2,000; Dako, Carpinteria, CA, USA), synaptophysin (dilution, 1:75; Dako) and Ki-67 (dilution, 1:50; Thermo Scientific Lab Vision) were performed. Tissue samples were fixed in $10 \%$ formaldehyde overnight, dehydrated and embedded in paraffin. Sections $(5 \mathrm{~mm})$ were stained with $\mathrm{HE}$ and also used for immunohistochemistry.

The following characteristics were collected for each patient: Age, gender, presenting symptoms, location of primary tumor, type of surgery, complications, pathological features, including tumor size, mitotic count and Ki-67 index, Tumor-Node-Metastasis (TNM) stage (15), adjuvant therapy, the patient's disease status (13) at the last hospital visit and the most recent follow-up information.

For the determination of disease stage, the PNETs were classified into the localized, regional and distant groups. A localized PNET was defined as an invasive tumor that was completely confined to the organ of origin. A regional PNET was defined as a tumor with extension further than the limits of the organ of origin directly into the surrounding organs or tissue, or the involved regional lymph nodes, or a tumor that fulfilled each of the aforementioned criteria. Furthermore, a distant PNET was defined as a tumor that had spread to regions of the body that were remote from the primary tumor (16).

The present study was approved by the Institutional Review Boards of the First Affiliated Hospital, Zhejiang University School of Medicine, and informed consent was obtained from the patient for the publication of this report and any accompanying images.

Statistical analysis. Results are presented as median (range) and all statistical analyses were performed using SPSS 16.0 (SPSS, Inc., Chicago, IL, USA) for Windows. Overall survival (OS) was defined as the time between the initial diagnosis and mortality from any cause or to the time of the last known
Table I. Clinical and pathological characteristics for patients $(n=104)$.

\begin{tabular}{lc}
\hline Variable & Value \\
\hline Age, years & \\
Median & 52 \\
Range & $19-76$ \\
Gender, n (\%) & \\
Male & $49(47.1)$ \\
Female & $55(52.9)$ \\
Presentation, n (\%) & \\
Abdominal pain & $31(29.8)$ \\
Incidental finding & $30(28.8)$ \\
Abdominal discomfort & $9(8.7)$ \\
Jaundice & $4(3.8)$ \\
Hypoglycemia & $28(26.9)$ \\
Diarrhea & $2(1.9)$ \\
Tumor size, cm & \\
Median & 3 \\
Range & $0.8-19$ \\
Location, n (\%) & \\
Head/uncinate & $49(47.1)$ \\
Body & $17(16.3)$ \\
Tail & $38(36.5)$ \\
Sugical a &
\end{tabular}

Surgical approaches, $\mathrm{n}(\%)$

$\mathrm{R} 0$ resection

$\mathrm{DP}$

PD

LP

$17(16.3)$

$\mathrm{R} 1$ resection

Stage, n $(\%)$

Local

Regional

Distant

Grade, n (\%)

1

$47(45.2)$

2

$50(48.1)$

7 (6.7)

Distant metastasis, n (\%)

At initial diagnosis

During follow-up

Lymph node metastasis, n (\%)

Negative

Positive

$20(19.2)$

DP, distal pancreatectomy; PD, pancreaticoduodenectomy; LP, local resection of pancreatic tumor.

contact. Disease-free survival (DFS) was defined as the time between surgery and PNET recurrence. Those individuals who did not exhibit evidence of local recurrence or metastasis at the last follow-up and those patients who succumbed from causes that were unrelated to PNETs were censored in the analysis 

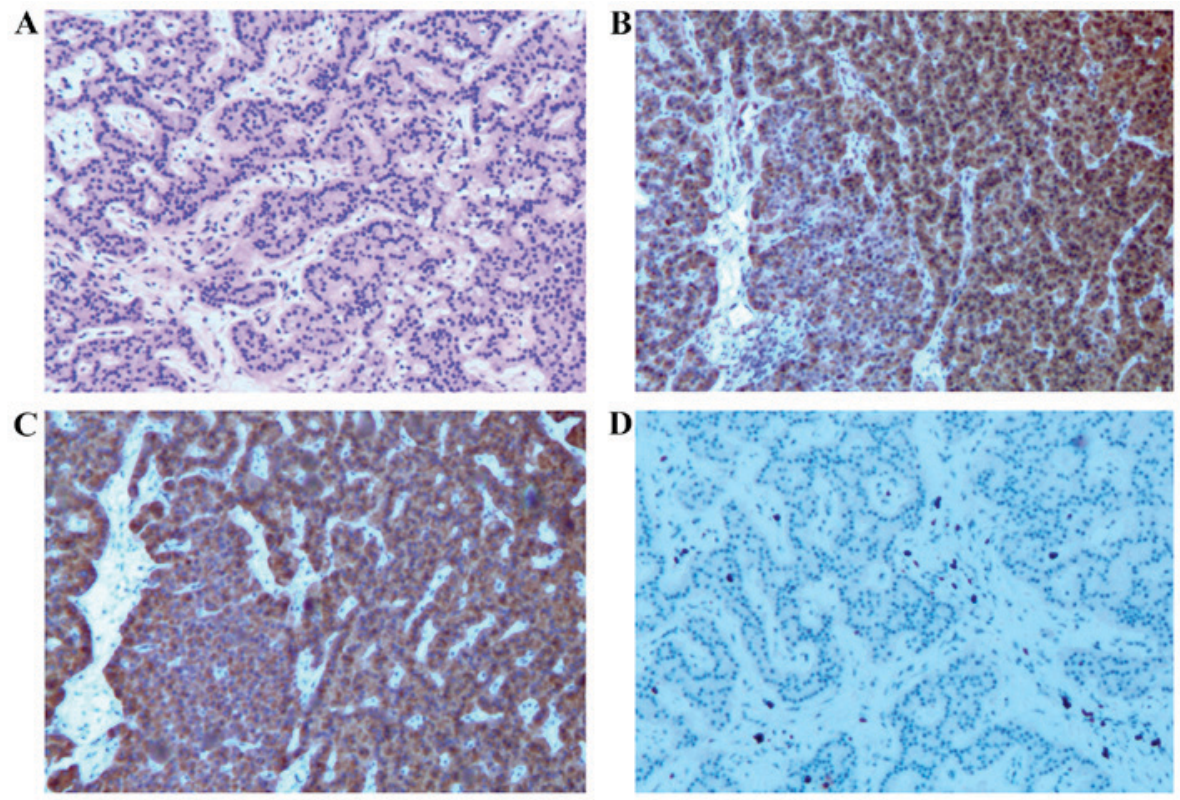

Figure 1. Pancreatic mass (x50 magnification). (A) Hematoxylin and eosin section, and immunohistochemistry showing marked positivity for (B) chromogranin A and (C) synaptophysin, and positivity for (D) Ki-67.

of DFS rate. OS and DFS rate analyses were performed by Kaplan-Meier methodology with log-rank testing. Cox proportional hazard models were used to estimate hazard ratios for OS and DFS rates, and to determine independent risk factors. All tests were two-sided, with $\mathrm{P}<0.05$ considered to indicate a statistically significant difference.

\section{Results}

Clinicopathological characteristics of PNETs. Among the 104 Chinese patients with PNETs, 49 (47.1\%) were men and $55(52.9 \%)$ were women. The median age at presentation was 52 years (range, 19-76 years). Of the 104 PNETs, 30 (28.8\%) were found incidentally during a health examination. The most common presentation of the symptomatic PNETs was abdominal pain in $31(29.8 \%)$ patients, followed by hypoglycemia in $28(26.9 \%)$, abdominal discomfort in $9(8.7 \%)$, jaundice in $4(3.8 \%)$ and diarrhea in $2(1.9 \%)$. The median size of the PNETs was $3 \mathrm{~cm}$ (range, $0.8-19.0 \mathrm{~cm}$ ). The PNETs were located in the head of the pancreas $(n=49 ; 47.1 \%)$, followed by the tail $(n=38$; $36.5 \%)$ and body $(n=17 ; 16.3 \%)$ (Table I). A total of 99 patients underwent curative resections (R0 resection, 95.2\%), in which the distal pancreatectomy was the most common procedure $(51.0 \%)$, followed by pancreaticoduodenectomy $(27.9 \%)$ and local resection of the pancreas $(16.3 \%)$. Palliative surgery (R1 resection, $4.8 \%$ ) was performed for only 5 patients, where symptomatic chemotherapy and somatostatin analog therapy were simultaneously performed.

The majority of tumors were of low or moderate grade (93.3\%, grade 1 or 2) and local or regional stage $(92.3 \%)$. In total, $8(7.7 \%)$ patients were classified as presenting with distant metastasis at the initial diagnosis, while 17 (17.3\%) patients presented with distant metastasis during the follow-up. The most frequent metastatic site was the liver. The patients received treatments that included radiofrequency ablation $(n=8)$, transarterial chemoembolization $(n=7)$, symptomatic chemotherapy $(\mathrm{n}=4)$, with intravenous infusion of $100 \mathrm{mg} / \mathrm{m}^{2}$ etoposide and $75 \mathrm{mg} / \mathrm{m}^{2}$ cisplatin daily for 1-3 days, repeated every 21 days, reoperation $(n=1)$ and somatostatin analog therapy $(n=2)$ with $20 \mathrm{mg}$ intramuscular Sandostatin LAR every 4 weeks for 3 months. The pathology showed lymph node metastasis in $20(19.2 \%)$ patients. Only 20 tumors (19.2\%) were functional, including 5 gastrinomas, 8 insulinomas, 4 glucagonomas and 3 cases of multiple endocrine neoplasia-1.

Natural course and prognostic factors of PNETs. The median duration of post-operative follow-up was 31 months. The 1, 3 and 5-year OS rates were 95, 85 and 73\%, respectively. Meanwhile, the 1,3 and 5-year DFS rates were 86, 72 and $68 \%$, respectively. The 5-year OS rates for grades 1 to 3 were 89.1, 63.6 and $43.8 \%(\mathrm{P}=0.002)$, while the 5-year OS rates for local, regional and distant disease stages were 90.4, 39.5 and $18.8 \%$ $(\mathrm{P}<0.001)$.

Fig. 2 illustrates that the OS and DFS rates were significantly different with regard to grade (grades 1, 2 and 3). Compared with grades 2 and 3, grade 1 PNET exhibited superior OS $(\mathrm{P}=0.002)$ and $\mathrm{DFS}(\mathrm{P}<0.001)$ rates. Fig. 3 illustrates that the OS and DFS rates were also significantly different with regard to stage (local, regional and distant disease) (all $\mathrm{P}<0.001)$. Compared with the regional or distant stage, PNET at the local stage exhibited superior OS and DFS rates. Furthermore, patients with a low Ki-67 index exhibited superior OS and DFS rates compared with patients with a high $\mathrm{Ki}-67$ index $(\mathrm{P}<0.001)$ (Fig. 4). The presence of lymph node metastasis was predictive of inferior OS and DFS rates compared with the absence of lymph node metastasis (Fig. 5). In addition, the OS rate for patients with PNETs was significantly decreased following $\mathrm{R} 1$ resection $(\mathrm{P}<0.001)$ (Fig. 6). In addition, when the PNET patients were divided into 2 groups by age using a cutoff value of 50 years, a significant difference in OS rate $(\mathrm{P}=0.033)$ was observed, whereas no statistical difference was observed in DFS rate $(\mathrm{P}=0.388)$. When the PNET patients were 
A

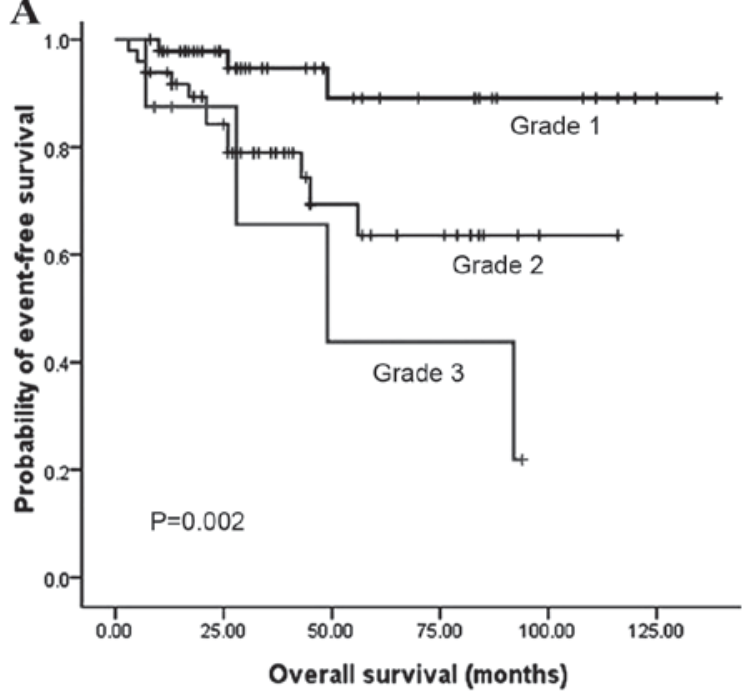

B

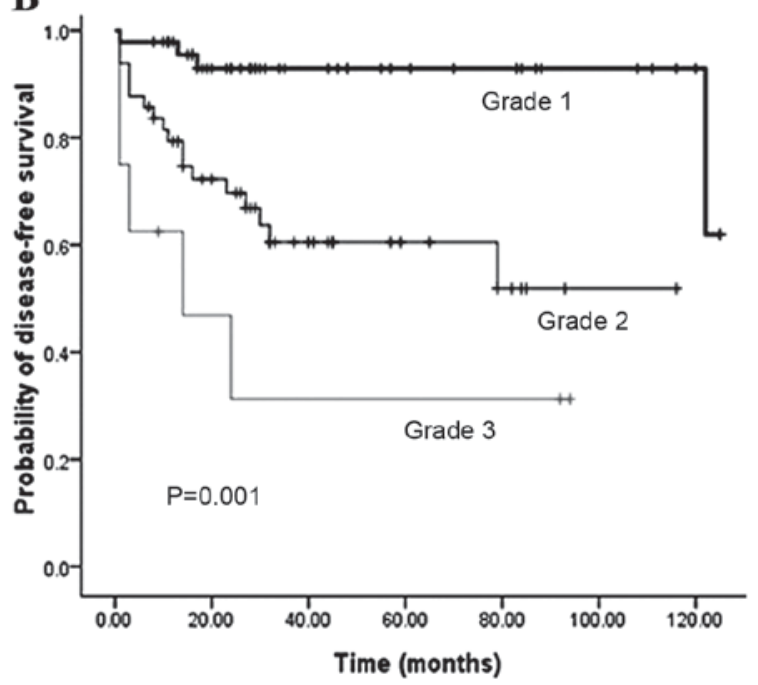

Figure 2. (A) Overall survival according to tumor grade and (B) disease-free survival according to tumor grade.

A

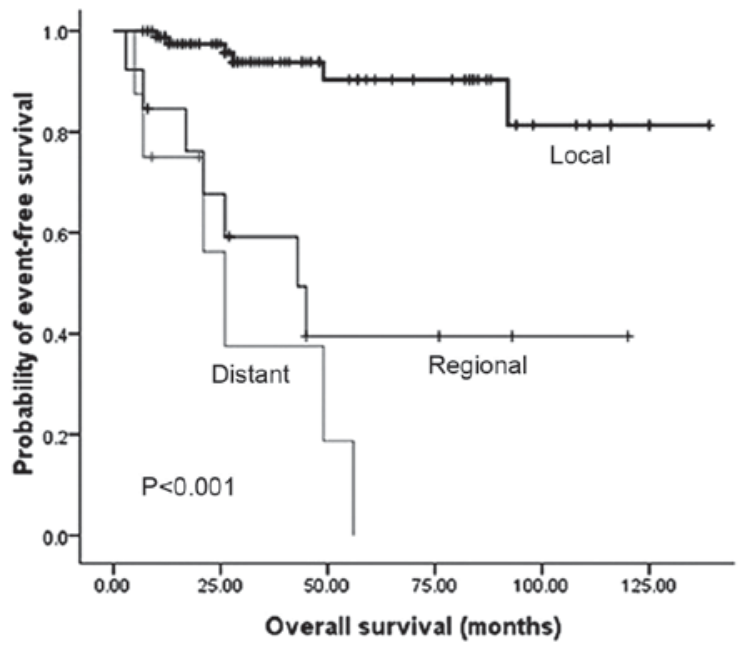

B

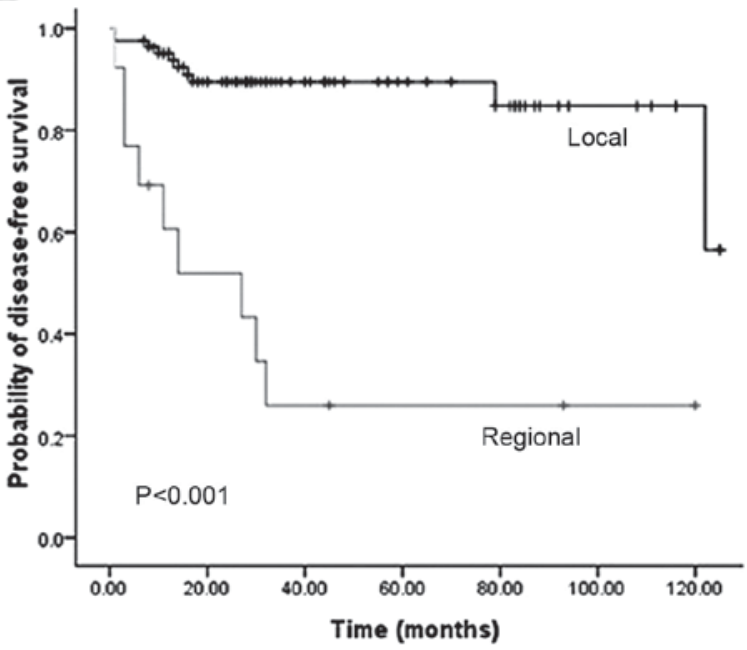

Figure 3. (A) Overall survival according to tumor stage and (B) disease-free survival according to tumor stage.

A

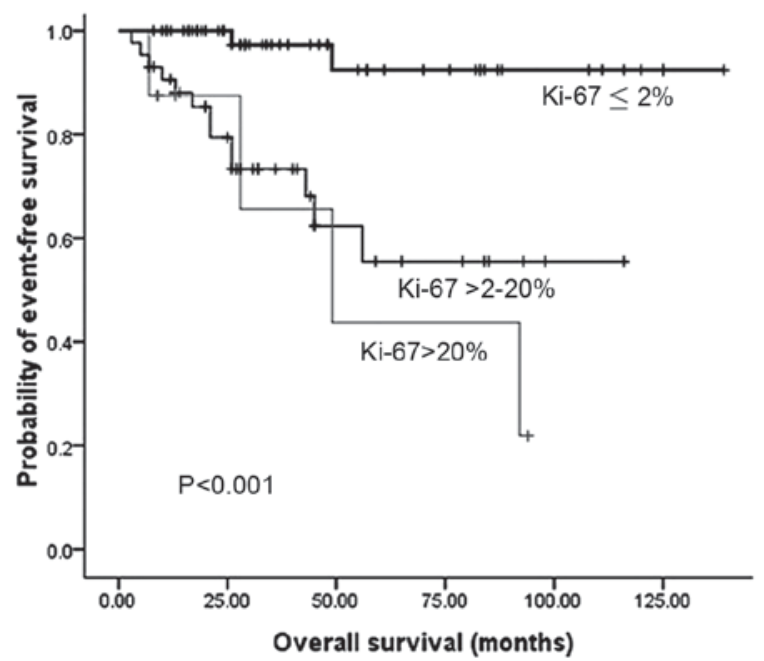

B

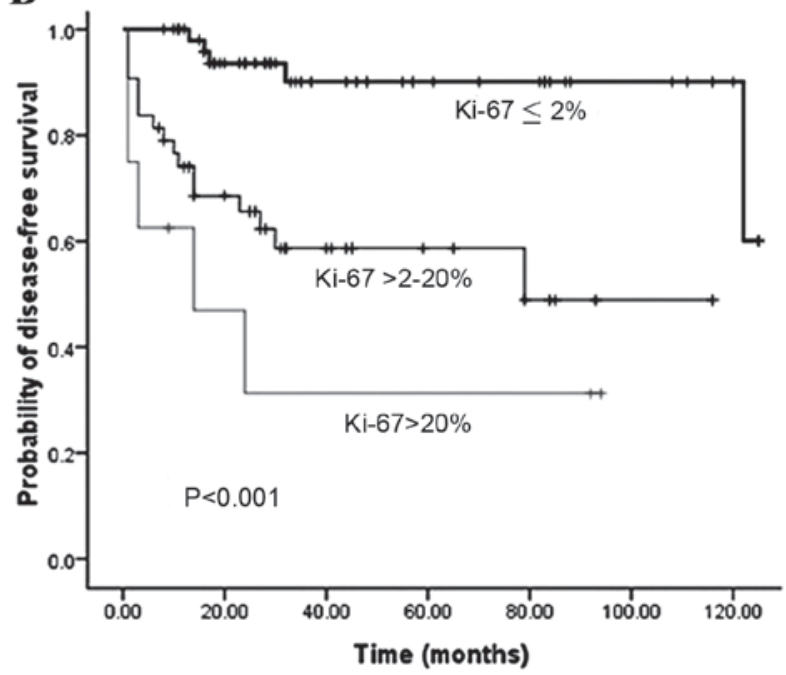

Figure 4. (A) Overall survival according to Ki-67 index and (B) disease-free survival according to Ki-67 index. 
A

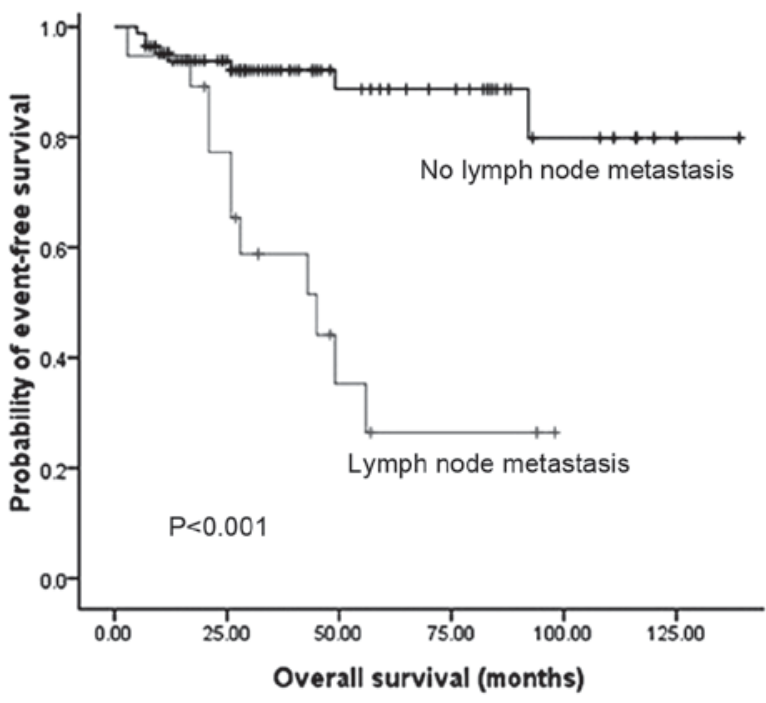

B

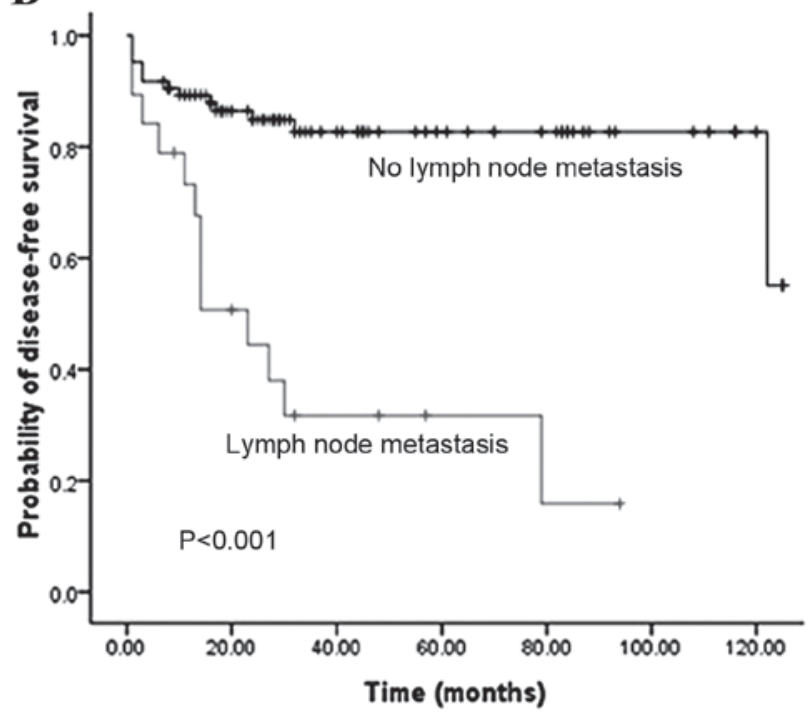

Figure 5. (A) Overall survival according to lymph node status and (B) disease-free survival according to lymph node status.

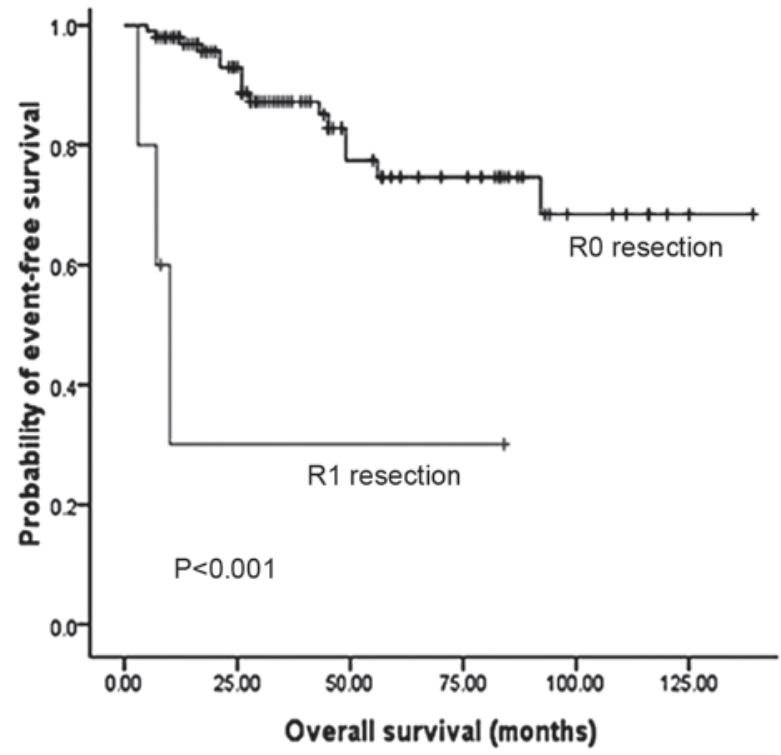

Figure 6. Overall survival according to resection margin status.

divided into the 2 groups of incidental diagnosis and symptomatic diagnosis, there was no statistical difference in OS or DFS rate. Compared with symptomatic PNETs, the incidental detection of PNETs was associated with longer OS (116 vs. 101 months; $\mathrm{P}=0.114)$ and DFS (103 vs. 86 months; $\mathrm{P}=0.146)$ times. DFS rate $(\mathrm{P}=0.010)$ was significantly different with regard to gender, whereas there was no significantly difference in OS rate $(\mathrm{P}=0.055)$. When the PNETs were divided into 3 groups according to primary tumor size, a significant difference in OS rate $(\mathrm{P}=0.044)$ was observed, whereas no statistical difference in DFS rate was observed, although it appeared that patients with smaller PNETs experienced longer DFS times than those with larger PNETs ( $\mathrm{P}=0.054)$ (Table II).

Multivariate $\mathrm{OS}$ rate variables of significance remaining in the final model included stage $(\mathrm{P}=0.02)$, grade $(\mathrm{P}=0.025)$, lymph node status $(\mathrm{P}<0.001)$, Ki-67 index $(\mathrm{P}=0.031)$ and surgical margin status $(\mathrm{P}<0.001)$ (Table III). Tumor size $(\mathrm{P}=0.044)$ and age $(\mathrm{P}=0.033)$ were those variables with a significant association with OS rate on univariate analysis, but no significant multivariate impact $(\mathrm{P}=0.254$ for tumor size; $\mathrm{P}=0.229$ for age). Meanwhile, upon multivariate analysis, stage $(\mathrm{P}=0.001)$, grade $(\mathrm{P}=0.017)$, lymph nodes status $(\mathrm{P}=0.02)$ and $\mathrm{Ki}-67$ index $(\mathrm{P}=0.016)$ were prognostic factors for DFS rate (Table IV).

\section{Discussion}

PNETs, a group of endocrine tumors arising in the pancreas, are rare and account for only $1-3 \%$ of all primary pancreatic malignancies (4). Several previous studies suggested that factors predicting survival following the resection of PNETs included age, gender, functional status of the tumor, pancreatic resection, lymph node involvement, distant metastases, grade of the tumor, and TNM stage, while others suggested that the presence or absence of cancer at the surgical margin, tumor location, tumor size and incidental or symptomatic tumors were the prognostic factors (6-14).

TNM stage and grade represent a simple and accurate instrument for mortality risk and disease-free assessment, as they accurately reflect the biology and natural history of the cancer. A study by Strosberg et al showed that the 5-year recurrence-free survival (RFS) rates for AJCC stages I, II and III were 78,53 and $33 \%$, respectively $(\mathrm{P}=0.01$ for stage I vs. stages II/III), which suggested that the American Joint Cancer Committee (AJCC) classifications for PNETs were prognostic for RFS (17). In addition, the use of the International Union for Cancer Control/AJCC/World Health Organization 2010 TNM staging results as an independent predictor of survival upon multivariable analysis suggests that measuring the extent of the cancer is relevant itself, as confirmed by a single series investigation in 2011 (18). Yang et al also indicated that the European Neuroendocrine Tumor Society TNM staging system may be superior in clinical practice when compared with the AJCC Staging Manual (seventh edition) for use in PNETs. Tumor grade, 
Table II. Univariate analyses of OS and DFS.

\begin{tabular}{|c|c|c|c|c|c|c|}
\hline Characteristics & $\begin{array}{l}\text { OS, } \\
\text { months }\end{array}$ & $\begin{array}{c}\text { 5-year survival } \\
\text { rate, } \%\end{array}$ & P-value & $\begin{array}{l}\text { DFS, } \\
\text { months }\end{array}$ & $\begin{array}{c}5 \text {-year } \\
\text { DFS rate, } \%\end{array}$ & P-value \\
\hline \multicolumn{7}{|l|}{ Age, years } \\
\hline$<50$ & 121.0 & 81.0 & \multirow[t]{2}{*}{0.033} & 96.0 & 75.1 & \multirow[t]{2}{*}{0.388} \\
\hline$\geq 50$ & 90.0 & 67.7 & & 89.0 & 70.9 & \\
\hline \multicolumn{7}{|l|}{ Gender } \\
\hline Male & 88.0 & 64.3 & \multirow[t]{2}{*}{0.055} & 78.0 & 60.5 & \multirow[t]{2}{*}{0.010} \\
\hline Female & 119.0 & 81.5 & & 103.0 & 83.4 & \\
\hline \multicolumn{7}{|l|}{ Incidental diagnosis } \\
\hline Yes & 116.0 & 91.8 & \multirow[t]{2}{*}{0.114} & 103.0 & 87.4 & \multirow[t]{2}{*}{0.146} \\
\hline No (symptomatic) & 101.0 & 68.2 & & 86.0 & 67.2 & \\
\hline \multicolumn{7}{|l|}{ Tumor grade } \\
\hline Grade 1 & 127.0 & 89.1 & \multirow[t]{3}{*}{0.002} & 116.0 & 92.9 & \multirow[t]{3}{*}{$<0.001$} \\
\hline Grade 2 & 85.0 & 63.6 & & 72.0 & 60.5 & \\
\hline Grade 3 & 58.0 & 43.8 & & 36.0 & 31.3 & \\
\hline \multicolumn{7}{|l|}{ Stage } \\
\hline Local & 124.0 & 90.4 & \multirow[t]{3}{*}{$<0.001$} & 111.0 & 89.5 & \multirow[t]{3}{*}{$<0.001$} \\
\hline Regional & 62.0 & 39.5 & & 42.0 & 26.0 & \\
\hline Distant & 30.0 & 18.8 & & NA & NA & \\
\hline \multicolumn{7}{|c|}{ Lymph node metastasis } \\
\hline Yes & 50.0 & 26.5 & \multirow[t]{2}{*}{$<0.001$} & 37.0 & 31.7 & \multirow[t]{2}{*}{$<0.001$} \\
\hline No & 121.0 & 88.7 & & 104.0 & 82.7 & \\
\hline \multicolumn{7}{|l|}{ Size } \\
\hline$\leq 2 \mathrm{~cm}$ & 121.0 & 87.0 & \multirow[t]{3}{*}{0.044} & 111.0 & 87.0 & \multirow[t]{3}{*}{0.054} \\
\hline$>2 \mathrm{~cm}$ to $4 \mathrm{~cm}$ & 90.0 & 73.2 & & 84.0 & 69.6 & \\
\hline$>4 \mathrm{~cm}$ & 87.0 & 74.0 & & 80.0 & 59.7 & \\
\hline \multicolumn{7}{|l|}{ Ki-67 index (\%) } \\
\hline $0-2$ & 131.0 & 92.3 & \multirow[t]{3}{*}{$<0.001$} & 114.0 & 90.1 & \multirow[t]{3}{*}{$<0.001$} \\
\hline$>2$ to 20 & 77.0 & 55.5 & & 69.0 & 58.7 & \\
\hline$>20$ & 58.0 & 43.8 & & 36.0 & 31.3 & \\
\hline \multicolumn{7}{|l|}{ Resection status } \\
\hline R0 & 109.0 & 74.7 & \multirow[t]{2}{*}{$<0.001$} & NA & NA & \multirow[t]{2}{*}{ NA } \\
\hline R1 & 30.0 & 30.0 & & NA & NA & \\
\hline
\end{tabular}

OS, overall survival; DFS, disease-free survival; NA, not available.

radical resection and the new AJCC system have all been confirmed as independent predictors for PNETs (13). In the present study, tumor stage was correlated with grade. The results showed that OS rate in the local disease stage was significantly higher compared with regional and distant disease stage $(\mathrm{P}<0.001)$, which was similar to the results found by Kim et al (16). Meanwhile, the present study also found that tumor grade was a critically important prognostic factor for $\mathrm{OS}$ rate $(\mathrm{P}=0.002)$. The present study documented that higher grade PNETs exhibited greater recurrence than PNETs of a lower grade $(\mathrm{P}<0.001)$. The most important factors to affect OS and DFS rates were stage and grade, as observed previously in a number of studies $(2,6,9,13)$. Greater recurrence was predicted in association with regional disease rather than local disease.
Certain studies, including the Surveillance, Epidemiology, and End Results report (19), showed that upon multivariate analysis, female gender was associated with a better prognosis, whereas in other previous studies, this was not observed (20). Moreover, women also experienced significantly longer DFS times compared with men. A study by Casadei et al appeared to suggest that factors (young age, female gender and the absence of comorbidities) represented a strong predictor of survival (21). In the present study, DFS rate $(\mathrm{P}=0.010)$ was found to be significantly different with regard to gender, whereas there was no significant difference in OS rate, although it appeared that women had longer OS times (119 vs. 88.0 months, $\mathrm{P}=0.055$ ). This result was similar to that of a study by Rindi et al, which found gender to be a prognostic factor for DFS in patients with neuroendocrine tumors (22). 
Table III. Cox regression analysis for overall survival.

\begin{tabular}{|c|c|c|c|}
\hline Variable & HR & $95 \% \mathrm{CI}$ & P-value \\
\hline \multicolumn{4}{|l|}{ Age } \\
\hline \multicolumn{4}{|l|}{$<50$} \\
\hline$\geq 50$ & 2.17 & $0.61-7.69$ & 0.229 \\
\hline \multicolumn{4}{|l|}{ Tumor grade } \\
\hline \multicolumn{4}{|l|}{ G1 } \\
\hline G2/G3 & 5.04 & $1.23-20.66$ & 0.025 \\
\hline \multicolumn{4}{|l|}{ Stage } \\
\hline \multicolumn{4}{|l|}{ Local } \\
\hline Regional/distant & 5.731 & $1.31-25.04$ & 0.020 \\
\hline \multicolumn{4}{|c|}{ Lymph node metastasis } \\
\hline \multicolumn{4}{|l|}{ No } \\
\hline Yes & 6.361 & 2.06-19.58 & $<0.001$ \\
\hline \multicolumn{4}{|l|}{$\mathrm{Ki}-67$ index, $\%$} \\
\hline \multicolumn{4}{|l|}{$\leq 2$} \\
\hline$>2$ & 4.13 & $1.14-15.02$ & 0.031 \\
\hline \multicolumn{4}{|l|}{ Resection status } \\
\hline \multicolumn{4}{|l|}{ R0 } \\
\hline $\mathrm{R} 1$ & 20.16 & $3.37-120.72$ & $<0.001$ \\
\hline
\end{tabular}

Table IV. Cox regression analysis for disease-free survival.

\begin{tabular}{|c|c|c|c|}
\hline Variable & HR & $95 \% \mathrm{CI}$ & P-value \\
\hline \multicolumn{4}{|l|}{ Gender } \\
\hline \multicolumn{4}{|l|}{ Female } \\
\hline Male & 2.11 & $0.83-5.40$ & 0.119 \\
\hline \multicolumn{4}{|c|}{ Tumor grade } \\
\hline \multicolumn{4}{|l|}{ G1 } \\
\hline G2/G3 & 4.25 & $1.30-13.92$ & 0.017 \\
\hline \multicolumn{4}{|l|}{ Stage } \\
\hline \multicolumn{4}{|l|}{ Local } \\
\hline Regional & 5.71 & $2.14-15.21$ & 0.001 \\
\hline \multicolumn{4}{|c|}{ Lymph node metastasis } \\
\hline \multicolumn{4}{|l|}{ No } \\
\hline Yes & 3.85 & $1.23-11.98$ & 0.020 \\
\hline \multicolumn{4}{|c|}{ Ki-67 index, \% } \\
\hline \multicolumn{4}{|l|}{$\leq 2$} \\
\hline$>2$ & 3.22 & $1.92-8.27$ & 0.016 \\
\hline
\end{tabular}

HR, hazard ratio; CI, confidence interval.

Possible explanations for these differences in tumor biology and survival may be gender imbalances for risk factors such as smoking, alcohol consumption, a different constitution or hormonal effects.

It is known that tumor size is an important prognostic factor in PNETs (23). In the present study, a smaller tumor size was associated with a longer OS time. Although tumor size was not significantly associated with DFS, patients with smaller PNETs experienced longer DFS times. In addition, age was an important prognostic factor for OS rate in the present study $(\mathrm{P}=0.033)$, as has been found in one previous study (19), but in contrast to other study results $(6,13)$.

Another factor potentially associated with adverse survival is lymph invasion (6). In the current study, the presence of lymph node metastasis was predictive of inferior OS and DFS rates compared with the absence of lymph node metastasis. This was supported by the studies of Scarpa et al (24) and Ito et al (25), which also identified lymph node metastasis as a relevant prognostic factor. By contrast, Bahra et al reported that lymph node metastases was not significant in determining the survival of non-functional PNETs, and a multivariate analysis of nodal stage revealed no significant differences with regard to the predicted cumulative survival probability $(\mathrm{P}=0.81)$ (26).

As a marker of cellular proliferation, Ki-67 has prognostic value in a number of malignancies, including PNETs $(26,27)$. Mitotic grade and $\mathrm{Ki}-67$ grade have each been used to distinguish between patients with a good prognosis and those with a poor prognosis. The univariate analysis in the study by Liu et al showed that mitotic count, Ki-67 index and tumor necrosis were all significant prognostic factors for regional well-differentiated PNETs (28). Another study also demonstrated that the predictive power of Ki-67 was enhanced when combined with metastasis status and tumor size (29). Cherenfant et al reported that a cut-off value of $>3 \%$ for $\mathrm{Ki}-67$ was the best mortality predictor $(83 \%)$, with an area under the curve of 0.85 . This Ki-67 value was also predictive of distant metastasis occurrence, with odds ratio and $95 \%$ confidence interval values of 9.22 and 1.55-54.55, respectively $(\mathrm{P}<0.015)(27)$. Scarpa et al observed no difference in outcome between those individuals with a Ki-67 value of $\leq 2 \%$ and those with a value of $\leq 5 \%$. In this study, the statistically significant cut-off value for Ki-67 was 5\%. A Ki-67 index of 5-20\% was associated with a 2-times higher risk of mortality from disease compared with a Ki-67 of $\leq 5 \%$. Patients with a Ki-67 of $>20 \%$ were 11 times more likely to succumb to disease compared with patients with a Ki-67 of $\leq 5 \%$ (24). In the present study, patients with a Ki-67 of $>2 \%$ showed significantly decreased OS and DFS rates upon multivariate analysis $(\mathrm{P}<0.05)$, which was similar to the results by Bahra et al $(26)$ and La Rosa et al (30). As these large variations exist in the Ki-67 index values in PNETs, a requirement exists for a larger study or a meta-analysis in order to identify the ideal cut-off point.

The risk of progression and mortality for incidentally diagnosed PNETs appears to be lower than patients who are symptomatic from their tumors at diagnosis. In the present study, there was no statistical difference between the incidental diagnosis and the symptomatic diagnosis with regard to OS $(\mathrm{P}=0.114)$ and DFS $(\mathrm{P}=0.146)$ rates. Cheema et al reported an $86 \%$ 5-year progression-free survival rate for incidentally diagnosed tumors compared with a 59\% 5-year progression-free survival rate for symptomatic tumors $(\mathrm{P}=0.007)$. Upon multivariate analysis, the strongest prognostic factor for progression was the incidental detection of the tumors (31).

In conclusion, the results of the present study suggested that stage, grade, Ki-67 index and lymph nodes involvement 
are significant prognostic factors for OS and DFS rates in PNETs. Furthermore, surgical margin status may also be an independent predictor for the prognosis of PNETs. These findings may serve as future useful clinical survival predictors, particularly with regard to resected disease.

\section{Acknowledgements}

This study was funded by the Projects of the Ministry of Public Health (grant no. 201002004).

\section{References}

1. Klimstra DS, Modlin IR, Coppola D, Lloyd RV and Suster S: The pathologic classification of neuroendocrine tumors: A review of nomenclature, grading, and staging systems. Pancreas 39: 707-712, 2010.

2. Fischer L, Kleeff J, Esposito I, Hinz U, Zimmermann A, Friess H and Büchler MW: Clinical outcome and long-term survival in 118 consecutive patients with neuroendocrine tumours of the pancreas. Br J Surg 95: 627-635, 2008.

3. Niederle MB, Hackl M, Kaserer K and Niederle B: Gastroenteropancreatic neuroendocrine tumours: The current incidence and staging based on the WHO and European Neuroendocrine Tumour Society classification: An analysis based on prospectively collected parameters. Endocr Relat Cancer 17: 909-918, 2010 .

4. Bilimoria KY, Talamonti MS, Tomlinson JS, Stewart AK, Winchester DP, Ko CY and Bentrem DJ: Prognostic score predicting survival after resection of pancreatic neuroendocrine tumors: Analysis of 3851 patients. Ann Surg 247: 490-500, 2008

5. Hauso O, Gustafsson BI, Kidd M, Waldum HL, Drozdov I, Chan AK and Modlin IM: Neuroendocrine tumor epidemiology: Contrasting Norway and North America. Cancer 113: 2655-2664, 2008

6. Yang M, Zeng L, Zhang Y, Su AP, Yue PJ and Tian BL: Surgical treatment and clinical outcome of nonfunctional pancreatic neuroendocrine tumors: A 14-year experience from one single center. Medicine (Baltimore) 93: e94, 2014

7. Centonze DC, Cinardi N and Giannone G: Surgical resection for neuroendocrine tumors of the pancreas: A fourteen years single institutional observation. Eur Rev Med Pharmacol Sci 18 (Suppl 2): 32-35, 2014.

8. Wang SE, Su CH, Kuo YJ, Shyr YM, Li AF, Chen TH, Wu CW and Lee $\mathrm{CH}$ : Comparison of functional and nonfunctional neuroendocrine tumors in the pancreas and peripancreatic region. Pancreas 40: 253-259, 2011.

9. Casadei R, Ricci C, Pezzilli R, Campana D, Tomassetti P, Calculli L, Santini D, Antonacci N and Minni F: Value of both WHO and TNM classification systems for patients with pancreatic endocrine tumors: Results of a single-center series. World J Surg 33: 2458-2463, 2009.

10. Casadei R, Ricci C, Rega D, D'Ambra M, Pezzilli R, Campana D, Nori F, Minni F and Tomassetti P: Pancreatic endocrine tumors less than $4 \mathrm{~cm}$ in diameter: Resect or enucleate? A single-center experience. Pancreas 39: 825-828, 2010.

11. Franko J, Feng W, Yip L, Genovese E and Moser AJ: Non-functional neuroendocrine carcinoma of the pancreas: Incidence, tumor biology, and outcomes in 2,158 patients. J Gastrointest Surg 14: 541-548, 2010.

12. Casadei R, Ricci C, Pezzilli R, Campana D, Tomassetti P, Calculli L, Santini D, D'Ambra M and Minni F: Are there prognostic factors related to recurrence in pancreatic endocrine tumors? Pancreatology 10: 33-38, 2010.

13. Yang M, Zeng L, Zhang Y, Wang WG, Wang L, Ke NW, Liu XB and Tian BL: TNM staging of pancreatic neuroendocrine tumors: An observational analysis and comparison by both AJCC and ENETS systems from 1 single institution. Medicine (Baltimore) 94: e660, 2015.

14. Partelli S, Inama M, Rinke A, Begum N, Valente R, Fendrich V, Tamburrino D, Keck T, Caplin ME, Bartsch D, et al: Long-term outcomes of surgical management of pancreatic neuroendocrine tumors with synchronous liver metastases. Neuroendocrinology 102: 68-76, 2015.
15. Rindi G, Klöppel G, Alhman H, Caplin M, Couvelard A, de Herder WW, Erikssson B, Falchetti A, Falconi M, Komminoth $\mathrm{P}$, et al: TNM staging of foregut (neuro)endocrine tumors: A consensus proposal including a grading system. Virchows Arch 449: 395-401, 2006.

16. Kim SJ, Kim JW, Oh DY, Han SW, Lee SH, Kim DW, Im SA, Kim TY, Heo DS and Bang YJ: Clinical course of neuroendocrine tumors with different origins (the pancreas, gastrointestinal tract, and lung). Am J Clin Oncol 35: 549-556, 2012.

17. Strosberg JR, Cheema A, Weber JM, Ghayouri M, Han G, Hodul PJ and Kvols LK: Relapse-free survival in patients with nonmetastatic, surgically resected pancreatic neuroendocrine tumors: An analysis of the AJCC and ENETS staging classifications. Ann Surg 256: 321-325, 2012.

18. Strosberg JR, Cheema A, Weber J, Han G, Coppola D and Kvols LK: Prognostic validity of a novel American Joint Committee on Cancer Staging Classification for pancreatic neuroendocrine tumors. J Clin Oncol 29: 3044-3049, 2011.

19. Yao JC, Hassan M, Phan A, Dagohoy C, Leary C, Mares JE, Abdalla EK, Fleming JB, Vauthey JN, Rashid A and Evans DB: One hundred years after 'carcinoid': Epidemiology of and prognostic factors for neuroendocrine tumors in 35,825 cases in the United States. J Clin Oncol 26: 3063-72, 2008

20. Strosberg J, Gardner N and Kvols L: Survival and prognostic factor analysis of 146 metastatic neuroendocrine tumors of the mid-gut. Neuroendocrinology 89: 471-476, 2009.

21. Casadei R, Ricci C, Tomassetti P, Campana D and Minni F: Factors related to long-term survival in patients affected by well-differentiated endocrine tumors of the pancreas. ISRN Surg 2012: 389385, 2012.

22. Rindi G, Falconi M, Klersy C, Albarello L, Boninsegna L, Buchler MW, Capella C,Caplin M, Couvelard A, Doglioni C, et al: TNM staging of neoplasms of the endocrine pancreas: Results from a large international cohort study. J Natl Cancer Inst 104: 764-77, 2012

23. Bettini R, Boninsegna L, Mantovani W, Capelli P, Bassi C, Pederzoli P, Delle Fave GF, Panzuto F, Scarpa A and Falconi M: Prognostic factors at diagnosis and value of WHO classification in a mono-institutional series of 180 non-functioning pancreatic endocrine tumours. Ann Oncol 19: 903-908, 2008.

24. Scarpa A, Mantovani W, Capelli P, Beghelli S, Boninsegna L, Bettini R, Panzuto F, Pederzoli P, delle Fave G and Falconi M: Pancreatic endocrine tumors: Improved TNM staging and histopathological grading permit a clinically efficient prognostic stratification of patients. Mod Pathol 23: 824-833, 2010.

25. Ito H, Abramson M, Ito K, Swanson E, Cho N, Ruan DT, Swanson RS and Whang EE: Surgery and staging of pancreatic neuroendocrine tumors: A 14-year experience. J Gastrointest Surg 14: 891-898, 2010.

26. Bahra M, Jacob D, Pascher A, Plockinger U, Kristiansen G, Neuhaus P and Langrehr JM: Surgical strategies and predictors of outcome for malignant neuroendocrine tumors of the pancreas. J Gastroenterol Hepatol 22: 930-935, 2007.

27. Cherenfant J, Talamonti MS, Hall CR, Thurow TA, Gage MK, Stocker SJ, Lapin B, Wang E, Silverstein JC, Mangold K, et al: Comparison of tumor markers for predicting outcomes after resection of nonfunctioning pancreatic neuroendocrine tumors. Surgery 156: 1504-1511, 2014.

28. Liu TC, Hamilton N, Hawkins W, Gao F and Cao D: Comparison of WHO Classifications (2004, 2010), the Hochwald grading system, and AJCC and ENETS staging systems in predicting prognosis in locoregional well-differentiated pancreatic neuroendocrine tumors. Am J Surg Pathol 37: 853-859, 2013.

29. Ferrone CR, Tang LH, Tomlinson J, Gonen M, Hochwald SN, Brennan MF, Klimstra DS and Allen PJ: Determining prognosis in patients with pancreatic endocrine neoplasms: Can the WHO classification system be simplified? J Clin Oncol 25: 5609-5615, 2007.

30. La Rosa S, Rigoli E, Uccella S, Novario R and Capella C: Prognostic and biological significance of cytokeratin 19 in pancreatic endocrine tumours. Histopathology 50: 597-606, 2007.

31. Cheema A, Weber J and Strosberg JR: Incidental detection of pancreatic neuroendocrine tumors: An analysis of incidence and outcomes. Ann Surg Oncol 19: 2932-2936, 2012. 